\section{A reply to Dusoir}

\section{MICHAEL KUBOVY \\ Yale University, New Haven, Connecticut 06520}

Kubovy (1977) has argued that conservative placement of the criterion in signal detection may be due to a systematic misconception of the shape of the underlying distributions. The purpose of the present note is to show that none of the arguments advanced by Dusoir (1978) in his comments on the above paper refute Kubovy's conjecture, nor do they invalidate Kubovy's data.

\section{DUSOIR'S FIRST COMMENT}

Dusoir correctly points out that my application of Bayes' theorem (Kubovy, 1977, pp. 277-278) did not take prior odds into account. Because the paragraphs in question are unclear, here is an amended version, which better communicates the intended idea.

I wish to conjecture, as I did in the article to which Dusoir refers, that subjects place their criteria conservatively in signal detection because they misconceive the form of the stimulus distributions or the relationship between the stimulus distributions.

Most training in signal detection situations is done under symmetric payoffs and with equal a priori probabilities of noise and of signal plus noise. Consider, for example, an observer who is ideal in all respects but one: after training, he underestimates the area under the tails of the two stimulus distributions. In such a case, likelihood ratios greater than 1 would be overestimated, and likelihood ratios less than 1 would be underestimated. Similarly, in this situation, where a priori probabilities are equal, posterior probabilities greater than .5 would be overestimated, and posterior probabilities less than .5 would be underestimated. Because this training determines the subject's conception of the distributions, we will call the posterior probabilities learned in this situation underlying posterior probabilities. The pattern of probability estimates we have just described is a pattern of radical underlying posterior probabilities. It would have been just as correct to say that this pattern represented radical subjective

The data in this article come from the author's dissertation (Kubovy, 1971). The data collection was supported by grants from the Faculty of Social Science of the Hebrew University of Jerusalem and the Marshall Fund for Research in Social Psychology. The author's current work on decision making is supported by NSF Grant BNS77-00077 to Yale University. I am grateful to A.F. Healy for her comments on a draft of this reply. Reprints may be requested from Michael Kubovy, Department of Psychology, Yale University, Yale Station Box 11A, New Haven, Connecticut 06520 . likelihood ratios. However, it was decided to discuss underlying posterior probabilities because the judgments elicited in the experiment reported by Kubovy (1977) were judgments of posterior probabilities.

To see how radical underlying posterior probabilities lead to conservative criterion placement, consider the deficient observer described above in a signal detection situation with an a priori probability of signal plus noise of .25 , and a symmetric payoff matrix. Since the calculation of $\beta^{*}$ does not require any knowledge of the distributions, our deficient observer will do so correctly, choosing $\beta^{*}=3$. Because of the observer's radical subjective likelihood ratios, the observation judged by our observer to have a likelihood ratio $1(x)=3$ will, in fact, be less extreme, and the resultant criterion placement will be conservative.

In line with the above, the three figures in Kubovy (1977) plot posterior probabilities. In Figures 1 and 2 , the underlying posterior probability is inferred from the value of $\beta^{*}$, the likelihood ratio of the optimal cutoff point, and is plotted as a function of $\beta$, the likelihood ratio of the observed cutoff point. In Figure 3, posterior probability judgments elicited from subjects are plotted as a function of the true posterior probabilities of the judged observation.

To summarize my response to Dusoir's first point: The point is well taken, but the two relevant paragraphs in Kubovy (1977) are easily amended to capture the intention of the conjecture about conservatism without affecting any of the remaining parts of the argument.

\section{DUSOIR'S SECOND COMMENT}

Dusoir correctly points out that Kubovy (1977) erred in labeling the Green and Swets (1966/1974, p. 91) data. This observation, however, has no bearing on the argument Kubovy made regarding these data, namely that a discrepancy between the degree of conservatism in the values-variable and in the probabilities-variable conditions-if observed in temporal contiguity-would count as evidence against his conjecture about conservatism.

\section{DUSOIR'S THIRD COMMENT}

Dusoir presents approximate data calculated from Galanter and Holman (1967, Experiment 1) which show a discrepancy between the degree of conservatism in the values-variable and in the probabilitiesvariable conditions, and were collected in temporal contiguity (whereas the Green and Swets data discussed by Kubovy were not). I concur with Dusoir that there is some evidence for a discrepancy between 
the degree of conservatism in the two conditions, but only in one subject, and then in the direction opposite to the discrepancy found in Green and Swet's data.

I see no reason to treat the discrepancy more seriously with this evidence in hand than did Kubovy (1977). One subject shows a discrepancy in one direction, the other shows no discrepancy, and a third shows a discrepancy in the other direction. There is still reason for concern about these discrepancies, but they are not sufficiently compelling to force us to reject Kubovy's (1977) conjecture.

Dusoir misuses the verb to ignore, when he claims that Kubovy "ignored" the Galanter and Holman data. One can only ignore something of which one was aware, or perhaps one should have been aware. A glance at Dusoir's Footnote 1 will show that the Galanter and Holman data presented by Dusoir were not actually reported by Galanter and Holman. The data were extracted from the Galanter and Holman article by fitting curves by eye to Galanter and Holman's published plots, and then calculating the table entries from the points on the fitted line nearest to the original data points. Given the crudeness of their derivation, it may be appropriate to ignore these data.

\section{DUSOIR'S FOURTH COMMENT}

Dusoir claims that Kubovy "lumped together several related but nonequivalent models which need to be distinguished." He then says, "Kubovy's argument quoted in Section 2 above is valid only for model A." Model A is, in fact, the one intended in Kubovy (1977) and formulated more explicitly above. in response to Dusoir's first comment. Thus model B is no more than an implausible straw man derived by Dusoir.

Dusoir correctly argues that Kubovy's (1977) conjecture implies the independence of $d^{\prime}$ and $\beta$, and he then cites evidence to the effect that $d^{\prime}$ and $\beta$ are not independent. But Kubovy's conjecture was never designed to answer all the objections to the theory of signal detection interpreted as a descriptive theory. So, to reject Kubovy's conjecture because it does not accommodate a correlation between $d^{\prime}$ and $\beta$ is like refusing to repair the pocket of a jacket because it will still be missing a button after that repair. None of the four other explanations traditionally invoked to account for conservatism (reviewed by Kubovy, 1977) can handle correlations between $d^{\prime}$ and $\beta$, and thus all current explanations of conservatism are equally refuted by this evidence.

Dusoir's model $C$ is is his attempt to modify model $\mathrm{A}$ so as to be consistent with the observed correlation between $d^{\prime}$ and $\beta$. His model $D$ is a further modification designed to accommodate the Green and Swets (1966) and Galanter and Holman (1967) data as well. Although these models may achieve these goals, it is a matter of scientific strategy whether the assumptions of model A should be modified at ail, or whether some other aspect of signal detection theory should be modified in order to accommodate the data cited by Dusoir. Clearly, further research is needed.

\section{DUSOIR'S FIFTH COMMENT}

Dusoir argues that the pattern of results obtained on the even-numbered trials of Kubovy's (1971, 1977) experiment could be due to a reaction against the bias present in the previous trial's "influence" responses. He argues, furthermore, that during Sessions 7-10, an influence response was more likely to overestimate posterior probabilities greater than .5 , whereas the reverse was true for posterior probabilities less than .5 .

Two assumptions are made by Dusoir in this reinterpretation of Kubovy's experiment: (a) the subjects manifested delayed anticonformity; (b) the pattern of influence responses was systematically related to the data reported. Let us consider these assumptions individually.

\section{Anticonformity}

There was a significant amount of conformity on the influence trials. In order to demonstrate this point, some further description of the design of the experiment is called for. All the observations that occurred on influence trials also occurred on feedback trials; half of them came first on an influence trial and half of them came first on a feedback trial. Let a subject's response to an observation $\mathrm{x}$ on a feedback and an influence trial be $r(x, \emptyset)$ and $r(x, s)$, respectively, where $s$ is the influence response. Influence trials can be partitioned into conforming, anticonforming, independent, and irrelevant. A response will be conforming if $s>r(x, \emptyset)$ and $r(x, s)\rangle$ $r(x, \emptyset)$, or if $s<r(x, \emptyset)$ and $r(x, s)<r(x, \emptyset)$. A response will be anticonforming if $s>r(x, \emptyset)$ and $r(x, s)<r(x, \emptyset)$, or if $s<r(x, \emptyset)$, and $r(x, s)>r(x, \emptyset)$. A response will be independent if $s \neq r(x, \emptyset)$ and $r(x, s)=r(x, \emptyset)$. Finally, a response will be irrelevant if $s=r(x, \emptyset)$.

Consider the following index of conformity: $\mathrm{C}=\mathrm{N}(\mathrm{c}) /[\mathrm{N}(\mathrm{c})+\mathrm{N}(\mathrm{a})]$, where $\mathrm{N}(\mathrm{c})$ is the number of conforming trials, and $N(a)$ is the number of anticonforming trials. Were there no tendency to conform, we would expect $C=.5$. The 24 observed proportions ( 2 for each subject), calculated over pairs of sessions, ranged from .57 to .79 , and their median was .69. A chi-square test showed that all but one of these values were significantly different from .5 ( $p<.001$ for 24 values, and $p<.01$ for one).

Another way of establishing conformity is by analyzing the amount of shift rather than the proportion of trials on which shifts occurred. Let 

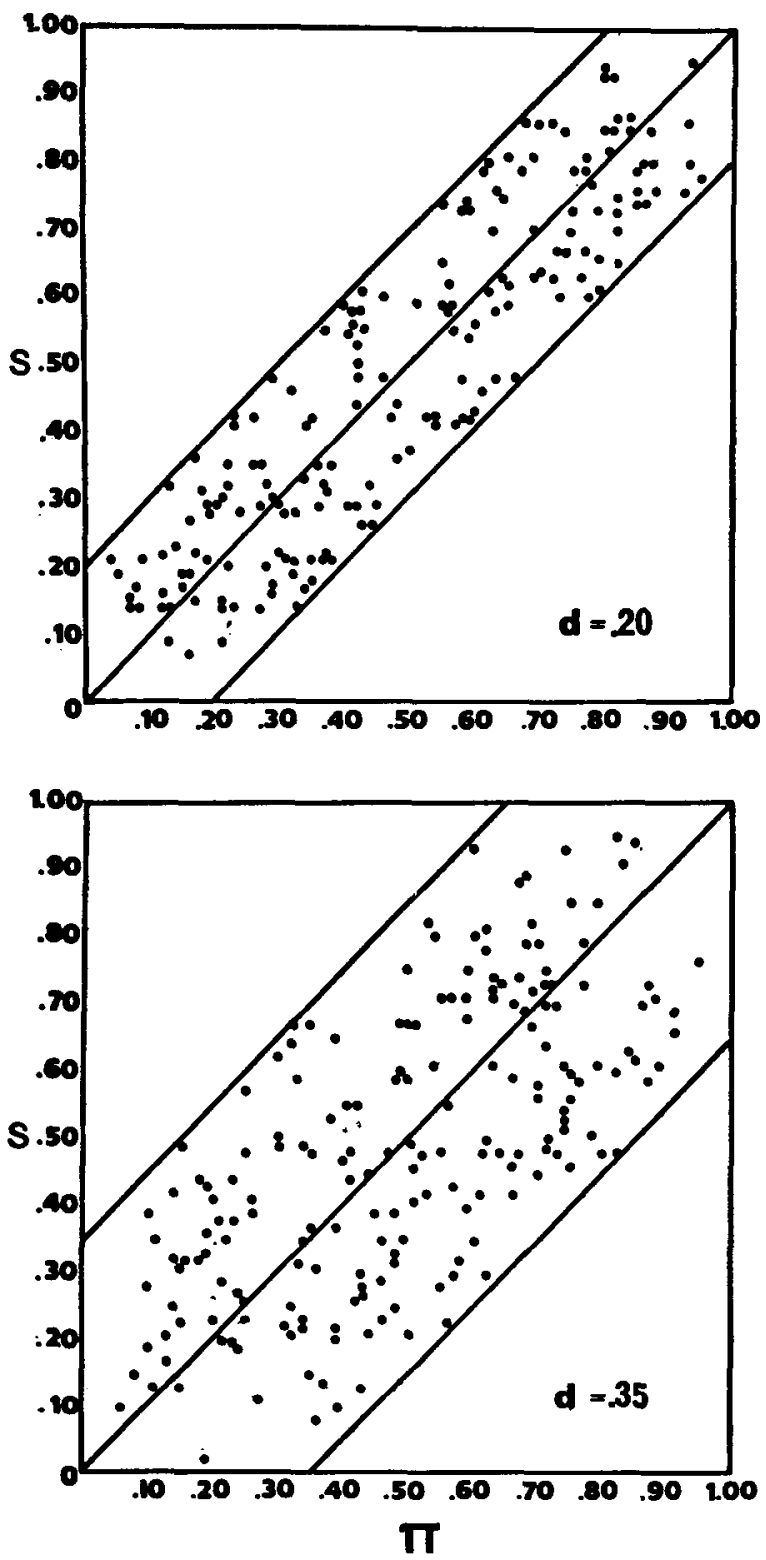

Figure 1. Influence response (s) as a function of the true posterior probability ( $\pi$ ) of the observation given on that trial.

$$
\delta= \begin{cases}r(x, s)-r(x, \emptyset) & \text { if } s>r(x, \emptyset) \\ r(x, \emptyset)-r(x, s) & \text { otherwise }\end{cases}
$$

Were there no tendency to conform, we would expect the mean of $\delta$ to be 0 or negative. The 24 observed values of $\delta$, calculated over pairs of sessions, were all positive. A two-tailed t test showed that all but two of these values were significantly different from 0 ( $p<.001$ for 17 values, $p<.01$ for 5 values).

Finally, it was asked whether $\delta$ was greater on conformity trials than $-\delta$ on anticonformity trials. In all but one case, the average shift was greater on conforming trials. A two-tailed t test showed that all but one of the 24 values were significantly different from $0(p<.001$ for 21 values, $p<.05$ for 2 values).

To summarize, there was no tendency for subjects to anticonform on influence trials; on the contrary, there is strong evidence that they conformed. It is thus quite unlikely that the subjects reacted against the bias present in the previous trials' influence responses. Furthermore, the experiment was designed so as to preclude a relation between successive observations on influence and feedback trials. It is therefore hard to imagine how a subject making a decision on a feedback trial could be influenced by the influence response given on the preceding trial.

\section{The Pattern of Influence Responses}

I have no quarrel with Dusoir's mathematics showing that there was a systematic relation between the influence response and the correct posterior probability. In fact, his calculations are confirmed in Figure 1, which plots the influence response (s) as a function of the true posterior probability of the observation given on the same trial $(\pi)$. However, there is no evidence of an S-shaped curve in these scatterplots, and thus it is hard to imagine a process whereby these influence responses would give rise to the systematic pattern of results shown by Kubovy's subjects, even if some sort of delayed anticonformity were operating. If taken in conjunction with the massive evidence that, far from being anticonformists, subjects actually conformed to the influence responses, these further details about the nature of Kubovy's stimuli should allay any fears concerning the interpretability of Kubovy's experiment.

\section{REFERENCES}

Dusorr, A. E. Kubovy on "A possible basis for conservatism in signal detection and probabilistic tasks": Some comments. Perception \& Psychophysics, 1978, 23, 542-545.

Galanter, E., \& Holman, G. L. Some invariances of the isosensitivity function and their implications for the utility function of money. Journal of Experimental Psychology, 1967, 73, 333-339.

Green, D. M., \& Swets, J. A. Signal detection theory and psychophysics. Huntington, N.Y: Krieger, 1974. (Originally published, 1966)

KUBovy, M. [Normative and informational aspects of social influence.] Unpublished doctoral dissertation, Hebrew University of Jerusalem, Israel, 1971.

Kubovy, M. A possible basis for conservatism in signal detection and probabilistic categorization tasks. Perception \& Psychophysics, 1977, 22, 277-281.

(Received for publication March 27, 1978; accepted April 3, 1978.) 